\title{
Utilizing Synergism between the Transverse Abdominal and Pelvic Floor Muscles at Different Postures in Nulliparous Women: A Randomized Case-Control Study
}

\author{
Tímea Molnár ${ }^{a}$ Andrea Domján ${ }^{a}$ Mónika Szűcs ${ }^{b}$ Andrea Surányi ${ }^{c}$ József Bódis ${ }^{d}$ \\ ${ }^{a}$ Faculty of Health Sciences and Social Studies, University of Szeged, Szeged, Hungary; ${ }^{\text {b}}$ Department of Medical Physics and \\ Informatics, University of Szeged, Szeged, Hungary; 'Department of Obstetrics and Gynaecology, University of Szeged,

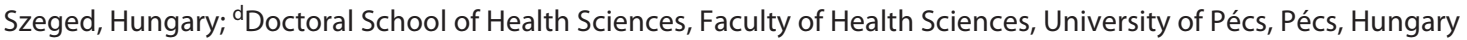

\section{Keywords}

Pelvic floor muscle training - Transverse abdominal muscle . Ultrasound measurement - Urinary incontinence - Vaginal surface electromyography

\begin{abstract}
Introduction: The aim of the study was to determine the effects of the pelvic floor muscle (PFM) training (PFM-T) in combination with transverse abdominal (TRA) muscle activation (CPFM-T) in female urinary incontinence. Methods: We enrolled nulliparous women in supine (SUG) $(n=22)$, sitting (SIG) $(n=19)$, and control (COG) $(n=14)$ groups. We conducted an 8-week cPFM-T programme. We examined the effect of training on the parameters with the Kruskal-Wallis test, the pairwise comparisons with the Mann-Whitney $U$ test, and the Wilcoxon rank test with the Bonferroni correction. Results: Before training, 15 participants reported occasional urinary leakage. After CPFM-T, 7 participants reported that urinary leakage had disappeared. Maximal isometric contraction of the PFMs until fatigue improved significantly in the SUG $(p<0.001)$ and SIG $(p=0.015)$ groups but not significantly in the COG group ( $p=0.499)$. Holding time increased in the SUG $(p=0.972)$ and the SIG $(p=0.717)$ groups and decreased in the COG group $(p=0.132)$. The dynamic endurance of the PFM improved significantly in the SUG group $(p<0.001)$ but not in the SIG $(p=0.798)$ and the COG
\end{abstract}

$(p=0.153)$ groups. The number of maximal fast contractions within 1 min increased in both the SUG $(p<0.001)$ and SIG $(p=0.813)$ groups and decreased in the COG group ( $p=$ $0.257)$. Relaxation improved significantly in the SIG group $(p=0.011)$. TRA mucle thickness increased in both training groups. Conclusion: Slow-twitch fibres of the PFM can be trained effectively with PFM-T in both the body positions.

(C) 2021 The Author(s)

Published by S. Karger AG, Basel

\section{Introduction}

Worldwide, an estimated $20-30 \%$ of young women have urinary incontinence (UI), making the frequency of this condition a fundamental problem [1]. According to Kegel, regular, specific strength training of the pelvic floor muscles (PFMs) has a beneficial effect on female UI and pelvic organ prolapse [2].

The effectiveness of Kegel's PFM-T exercises is classified by the International Consultation on Incontinence as level " $A$ " evidence in evidence-based medicine $[3,4]$. Several research groups have reported a co-contraction between the deep abdominal muscles and the PFM [5-8].

Andrea Surányi and József Bódis shared last authorship.

Tímea Molnár: Department of Physiotherapy, University of Szeged, Szeged, Hungary.
(C) 2021 The Author(s)

Published by S. Karger AG, Basel

This is an Open Access article licensed under the Creative Commons Attribution-NonCommercial-4.0 International License (CC BY-NC) (http://www.karger.com/Services/OpenAccessLicense), applicable to the online version of the article only. Usage and distribution for commercial purposes requires written permission.
Correspondence to:

Andrea Surányi, gaspar-suranyi.andrea@med.u-szeged.hu 
Several studies have revealed significantly higher resting PFM activity in unsupported sitting than supported sitting and in a standing posture than a supine position [8-11]. Chmielewska et al. [11] reported a significant, long-lasting contraction during unsupported sitting, due to the superior recruitment by the sitting posture of the sensorimotor control system to that by the supine position. Sufficient evidence that regular PFM-T improves the symptoms of incontinence has been reported $[3,12$, 13]. However, only a few studies support the effect of the coordination of the diaphragm, deep abdominal muscles, and PFM on incontinence $[14,15]$.

Here, we aimed to investigate whether - based on trunk muscle synergism - the condition and functioning of the PFM would improve in the sitting and supine postures or in the control group during PFM-T with forced exhalation. We hypothesized that the training position affects the muscle gymnastic performance.

\section{Materials and Methods}

\section{Participants}

We enrolled 58 healthy, young (mean \pm SD: $21.27 \pm 1.46$ years), nulliparous women in this trial. We recruited them through an online advertisement and then selected them for the training groups and the control group by T.M. We divided them into 2 study groups depending on the strength of the PFM and treated them with CPFM$T$ in both the supine and sitting positions. The assessment comprised 1 phase: 3 repetitions of sustained 5-s voluntary PFM contraction with 10-s relaxation. We calculated the mean of 3 contractions and set the obtained values in ascending order. The 22 participants with lower muscle strength (under $60 \mu \mathrm{V}$ ) comprised the SUG group and the 22 participants with higher muscle strength (over $60 \mu \mathrm{V}$ ) formed the SIG group. Only 19 participants however completed the programme in the SIG group. We enrolled these 19 participants with higher PFM tension (over $60 \mu \mathrm{V}$ ) in the SIG group because the PFM must be stronger to resist gravity [16]. We created a control group $(n=14)(\mathrm{COG})$ comprising 7 persons with a PFM tension under 60 $\mu \mathrm{V}$ and 7 individuals with a PFM tension over $60 \mu \mathrm{V}$. The COG did not change their lifestyles and did not undergo PFM-T. We included in the study groups participants willing to participate in the study and able to contract the pelvic floor and transverse abdominal (TRA) muscles correctly. Participants were required to maintain their everyday activities (attending lessons, sport activities, and so on). Exclusion criteria were known neurological or rheumatological diseases and previous vaginal or abdominal surgery.

\section{Subjective Measurements}

In the study, before and after the training programme, we used a self-administered questionnaire (online suppl. material File 1; see www.karger.com/doi/10.1159/000519590 for all online suppl. material), based on 3 validated questionnaires (the King's Health Questionnaire, the Incontinence Impact Questionnaire and the Urogenital Distress Inventory) [17]. We included in the questionnaire risk factors for UI (obstetric history, height and weight, stress, physical activity level, sport, vaginal and abdominal surgery) and questions relating to the urinary tract and anal canal (involuntary urinary leakage, cystitis, constipation) and sexual activity (orgasm problems).

\section{Objective Measurements}

Vaginal Surface Electromyography

We measured changes in PFM activity with a vaginal surface electromyographic (vsEMG) instrument (FemiScan ${ }^{\mathrm{TM}}$ MultiTrainer ${ }^{\mathrm{TM}}$; Mega Electronics, Kuopio, Finland), which measures the electronic signals of PFM activity, using a sterile Periform ${ }^{\mathrm{TM}}$ intravaginal probe with vsEMG electrodes [7]. A correct voluntary contraction of the PFM contributes to downward (posterior) movement of the indicator [18]. We positioned a reference electrode on the patients' left forearm.

\section{Procedure}

We tested the participants in a lying position with the hips and knees flexed, feet resting on a plinth, and knees supported to allow the hips and PFM to relax [7]. We instructed the volunteers on the proper placement of a FemiScan ${ }^{\mathrm{TM}}$ vsEMG probe in situ within the vagina. PFM activities were measured in a supine position twice (at 0 weeks and at 8 weeks). We displayed the vsEMG data as line graphs, thus providing visual feedback for the participants, and recorded the values in microvolts.

The participants performed 3 tasks:

1. PFM relaxation state for $30 \mathrm{~s}$;

2. Maximal isometric contraction till fatigue: 1 maximum voluntary tonic contraction of the PFM, held until fatigue, carried out once;

3. Dynamic endurance: fast, sudden maximum voluntary phasic contractions of the PFM, performed for $1 \mathrm{~min}$.

Transabdominal Ultrasound Measurement

We performed the TRA muscle measurements at the same time as the vaginal measurements and PFM tasks. We measured the degree of the change in the muscle thickness. We measured the thickness of the TRA muscle, assessing it by ultrasound visualization (Z.ONARE ${ }^{\mathrm{TM}} \mathrm{SP} /$ musculoskeletal, $8 \mathrm{MHz}$, 35-mm curved linear array transducer) on the lateral abdominal wall [19]. The participants were not allowed any movement of the hip and lumbar spine. We acquired the following recordings: (1) the thickness of the TRA muscle during PFM relaxation and (2) the TRA muscle thickness during maximal isometric contraction till PFM fatigue.

\section{Pelvic Floor Muscle Training}

A physical therapist (T.M.) supervised the training and carried out the evaluation. We also set up 1 control group $(n=14)$ (COG). We divided the participants into 2 study groups depending on the strength of the PFM and treated them with cPFM-T in both supine $(n=22)$ (SUG) and sitting $(n=19)(\mathrm{SIG})$ positions. The treatment for the SUG and the SIG comprised 8 sessions, with a 1-h cPFM-T session each week in a group and 15 min of individual home training, 6 times a week for a total of 8 weeks of treatment. Before the cPFM-T, we instructed all participants in the anatomy of the PFM and the lower urinary tract, the mechanism of continence, and unsupported sitting posture. We discouraged the slumped sitting posture since unsupported sitting postures require greater PFM activity than supported sitting postures [9]. All training sessions comprised warming up, gradual muscle strengthening, and relaxation exercises $[20,21]$. 
Table 1. Participant characteristics

\begin{tabular}{|c|c|c|c|c|}
\hline Age, years & $\begin{array}{l}21.00[20.00-22.00] \text { (SUG) } \\
21.00[20.00-22.00] \text { (SIG) } \\
21.50[21.00-23.00] \text { (COG) }\end{array}$ & & & \\
\hline Participants, $n$, (positions) & $\begin{array}{l}22 \text { (SUG) } \\
19(\mathrm{SIG}) \\
14(\mathrm{COG})\end{array}$ & & & \\
\hline $\mathrm{BMI}, \mathrm{kg} / \mathrm{m}^{2}$ & $\begin{array}{l}21.85[20.77-22.95] \text { (SUG) } \\
21.30[20.00-22.20] \text { (SIG) } \\
22.00[19.77-23.32] \text { (COG) }\end{array}$ & & & \\
\hline $\begin{array}{l}\text { Participants doing regular physical } \\
\text { activity more than twice a week, } n\end{array}$ & $\begin{array}{l}9(\mathrm{SUG}) \\
9(\mathrm{SIG}) \\
5(\mathrm{COG})\end{array}$ & & & \\
\hline BMI & $\operatorname{SUM}(n=55)$ & SUG $(n=22)$ & SIG $(n=19)$ & $\operatorname{COG}(n=14)$ \\
\hline $\begin{array}{l}\text { Normal BMI 18-24 } \\
\text { Overweight BMI } 25-29 \\
\text { Underweight BMI }<18\end{array}$ & $\begin{array}{l}45 \\
9 \\
1\end{array}$ & $\begin{array}{l}18 \\
3 \\
1\end{array}$ & $\begin{array}{l}16 \\
3 \\
0\end{array}$ & $\begin{array}{l}11 \\
3 \\
0\end{array}$ \\
\hline
\end{tabular}

Values are median [1st-3rd] quartiles for age and BMI and numbers of participants. BMI, body mass index; SUG, the supine study group; SIG, the sitting study group; COG, the control group.

Any woman can feel both TRA muscle and PFM activity at the medial anterior superior iliac spina (ASIS) because the initial intravaginal pressure is predominated by PFM activity and the later increase in pressure is produced by the combined activation of the PFM, the RA, the internal oblique muscle (IO), and the TRA muscle [22].

1. In the first 4 weeks of cPFM-T, all study groups $(n=41)$ did exercises in the supine position with the hip and knees flexed, feet resting on the plinth, a hip width apart.

2. In the second 4 weeks, the participants performed exercises, but while the SUG group $(n=22)$ did exercises in the supine position with the hips and knees flexed, feet resting on the plinth, the SIG group $(n=19)$ did exercises while sitting upright without support, with feet resting on the plinth.

\section{Statistical Analysis}

We examined the effect of training on the parameters with the Kruskal-Wallis test. We performed the pairwise comparisons with the Mann-Whitney U test and the Wilcoxon rank test with the Bonferroni correction. We performed all statistical analyses using $\mathrm{R}$ statistical program (version 3.5.1; R Foundation for R statistical computing). We considered values of $p<0.05$ to be statistically significant.

\section{Results}

\section{Questionnaire Results}

The participants were between 18 years and 25 years old, with a body mass index (BMI) of $19.77 \mathrm{~kg} / \mathrm{m}^{2}-23.32$ $\mathrm{kg} / \mathrm{m}^{2}$. Online supplementary material File 1 presents the questionnaire form, and we list the characteristics of participants in Table 1.

At the beginning of the training programme, 15 participants (4 SUG, 8 SIG, and 3 COG participants) complained of urinary leakage during stress (coughing). After the training programme, urinary leakage disappeared in 7 participants (3 SUG and 4 SIG participants), while the symptoms of the COG remained unchanged. All participants experienced the leakage of a few drops of urine but rarely urinary loss (first degree SI).

Women with a sexual partner $(n=31)$ reported positive changes in their sexual life after the training programme. Twenty participants (10 SUG, 8 SIG, and 2 COG participants) complained of air flow to the vagina in unusual positions. After the training programme, only 5 participants (2 SUG, 1 SIG, and 2 COG participants) reported unchanged conditions. Six participants (3 SUG and 3 SIG participants) suffered from constipation, which was in all cases resolved by the end of the training programme.

\section{Electromyographic and Ultrasound Results}

We present the data in Table 2 and Figure 1. The maximal isometric contraction of the PFM till fatigue improved significantly in both study groups; however, after cPFM-T, this increase was higher in the SUG group. The tonic contraction of the PFM improved in the COG group, but the 
Table 2. PFM activity while resting and during tonic and phasic contractions in COG, SIG, and the SUG groups as well as the thickness of TRA muscle during isometric contraction of PFM until fatigue and in a relaxed state

\begin{tabular}{|c|c|c|c|}
\hline & $\begin{array}{l}\text { Before training } \\
\text { median }\left[Q_{1}-Q_{3}\right]\end{array}$ & After training & $p$ value \\
\hline \multicolumn{4}{|c|}{ Maximal isometric contraction of PFM till fatigue (tonic), $\mu \mathrm{V}$} \\
\hline COG & $56.25[44.23-83.62]$ & $58.65[54.27-89.07]$ & 0.499 \\
\hline SIG & $91.20[63.55-124.20]$ & $115.60[84.50-131.80]$ & 0.015 \\
\hline SUG & $42.90[27.73-53.42]$ & $59.00[50.10-73.85]$ & $<0.001$ \\
\hline \multicolumn{4}{|c|}{ Holding time of PFM, s } \\
\hline COG & $21.25[14.58-27.50]$ & $17.00[12.50-19.41]$ & 0.132 \\
\hline SIG & $17.00[12.75-27.50]$ & $19.50[15.50-23.00]$ & 0.717 \\
\hline SUG & $19.50[16.50-29.75]$ & $21.25[13.25-28.62]$ & 0.972 \\
\hline \multicolumn{4}{|c|}{ Dynamic endurance of PFM (phasic), $\mu \mathrm{V}$} \\
\hline COG & $88.16[62.84-97.22]$ & $77.05[60.23-87.83]$ & 0.153 \\
\hline SIG & $122.00[95.30-142.20]$ & $115.16[94.68-128.42]$ & 0.798 \\
\hline SUG & $53.15[35.61-64.28]$ & $70.97[56.10-83.80]$ & $<0.001$ \\
\hline \multicolumn{4}{|c|}{ Repetitions of fast contractions of PFM (repetitions), $n$} \\
\hline COG & $33.00[29.25-36.00]$ & $31.00[29.25-33.75]$ & 0.257 \\
\hline SIG & $29.00[22.00-39.00]$ & $30.00[23.00-35.50]$ & 0.813 \\
\hline SUG & $26.00[21.75-33.50]$ & $32.00[27.25-39.75]$ & $<0.001$ \\
\hline \multicolumn{4}{|c|}{ Relaxation state of PFM, $\mu \mathrm{V}$} \\
\hline COG & $8.60[6.48-17.98]$ & $10.55[3.85-19.40]$ & 0.851 \\
\hline SIG & $21.40[8.35-27.05]$ & $12.40[3.00-19.45]$ & 0.011 \\
\hline SUG & $13.40[9.20-20.75]$ & $13.25[6.83-18.50]$ & 0.465 \\
\hline \multicolumn{4}{|c|}{ TRA muscle during relaxation state of PFM, $\mathrm{cm}$} \\
\hline COG & $0.38[0.33-0.44]$ & $0.31[0.27-0.34]$ & 0.209 \\
\hline SIG & $0.26[0.23-0.32]$ & $0.26[0.23-0.31]$ & 0.717 \\
\hline SUG & $0.28[0.26-0.31]$ & $0.27[0.25-0.31]$ & 0.422 \\
\hline \multicolumn{4}{|c|}{ TRA muscle during maximal isometric contraction of PFM till fatigue, $\mathrm{cm}$} \\
\hline COG & $0.63[0.61-0.74]$ & $0.49[0.45-0.57]$ & 0.007 \\
\hline SIG & $0.51[0.38-0.63]$ & $0.56[0.41-0.75]$ & 0.231 \\
\hline SUG & $0.53[0.44-0.73]$ & $0.58[0.46-0.68]$ & 0.570 \\
\hline \multicolumn{4}{|c|}{$\begin{array}{l}\text { Values are median [1st-3rd] quartiles for isometric contractions, resting of the PFM and the TRA muscle and } \\
\text { phasic contractions of the PFM. SUG, number of participants in the supine study group; SIG, number of participants } \\
\text { in the sitting study group; COG, number of participants in the control group; PFMs, pelvic floor muscles; TRA, trans- } \\
\text { verse abdominal. }\end{array}$} \\
\hline
\end{tabular}

improvement was not significant $(p=0.499)$. Although in both study groups, the holding time of the maximal isometric contraction of the PFM till fatigue and the thickness of the TRA muscle during the maximal isometric contraction of the PFM till fatigue both increased, these changes were not significant (Fig. 1b). The latter parameter decreased significantly in the COG (Fig. 1c; Table 2).

On the other hand, while the strength of the maximal fast contractions of the PFM within 1 min increased significantly in the SUG, it decreased in the SIG and the COG; this decrease however was not significant (Fig. 2a). In both study groups, the number of maximal fast contractions within a minute increased, but the increase was significant only in the SUG group. This parameter decreased in the COG group (Table 2; Fig. 2b).

Synergism between Abdominal and Pelvic Muscles
In both study groups, the values for relaxation of the PFM decreased, but the decrease was significant only in the SIG group $(p=0.011)$. This value increased in the COG group (Fig. 3a). The thickness of the TRA muscle during relaxation of the PFM decreased in the SUG group $(p=0.422)$ and the COG group $(p=0.209)$ but not in the SIG group $(p=0.717)$. Neither of these changes were significant (Fig. 3b; Table 2).

\section{Discussion}

Our study demonstrated that an 8-week period of cPFM-T with forced exhalation, performed by young nulliparous women in both the supine posture and the sitting 


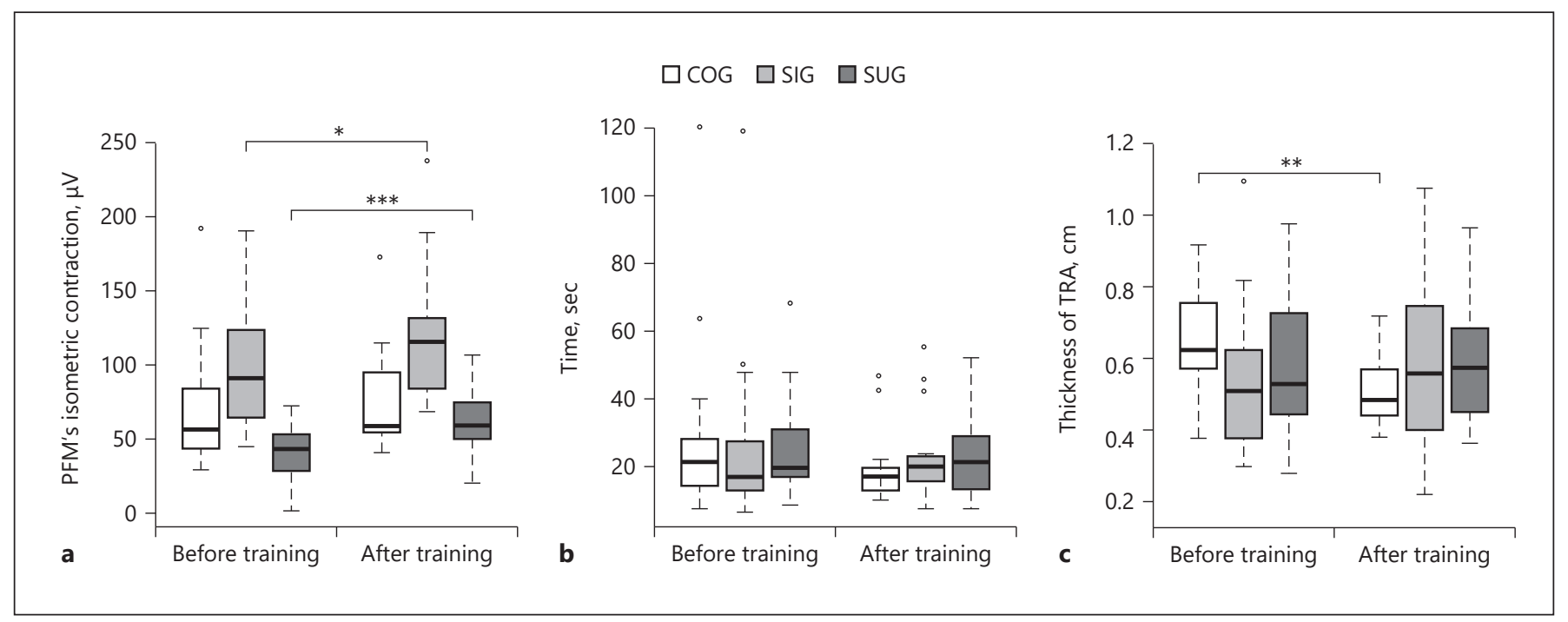

Fig. 1. vsEMG values of the maximal isometric contraction of PFM till fatigue (a); holding time of maximal isometric contraction of PFM till fatigue (b); thickness of TRA muscle during maximal isometric contraction of PFMs till fatigue (c). PFMs, pelvic floor muscles; TRA, transverse abdominal; vsEMG, vaginal surface electromyography; COG, the control group; SIG, the sitting study group; SUG, the supine study group.

Fig. 2. a vsEMG values of strength of maximal fast contractions within $1 \mathrm{~min}$. b Number of maximal fast contractions within 1 min. vsEMG, vaginal surface electromyography; COG, the control group; SIG, the sitting study group; SUG, the supine study group.

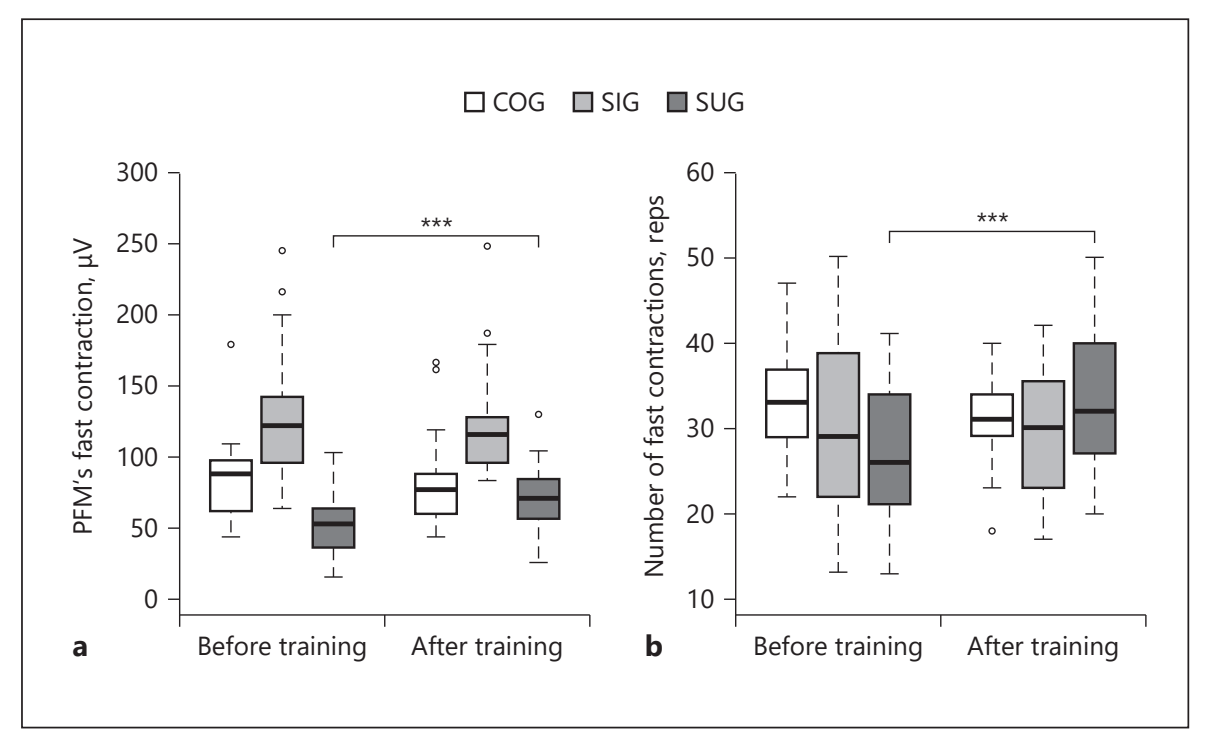

posture, using trunk muscle synergies, is effective in improving cases of incontinence. Furthermore, the holding period, rapid reaction and resting function of the PFM, and the thickness of TRA muscle improved due to both the modification of the body position and exhalation technique. Improvements in the holding function of the PFM were significant in both the supine position and the sitting position, but improvements in the rapid reaction of the PFM were significant only in the supine position, while those in the resting function of the PFM were sig- nificant only in the sitting position. The thickness of the TRA muscle improved in both the supine and sitting positions.

Assessments of the PFM can be used to determine which structural or functional features are deficient and to inform the design of subsequent training regimens to address these dysfunctions [24]. Sapsford [23] advocated a new approach to the rehabilitation of UI - motor relearning for diaphragmatic, deep abdominal muscles, and the PFM, rather than selective muscle strengthening. 
Fig. 3. a Relaxation values for PFM. b Thickness of TRA muscle during relaxation of PFM. PFMs, pelvic floor muscles; TRA, transverse abdominal; COG, the control group; SIG, the sitting study group; SUG, the supine study group.

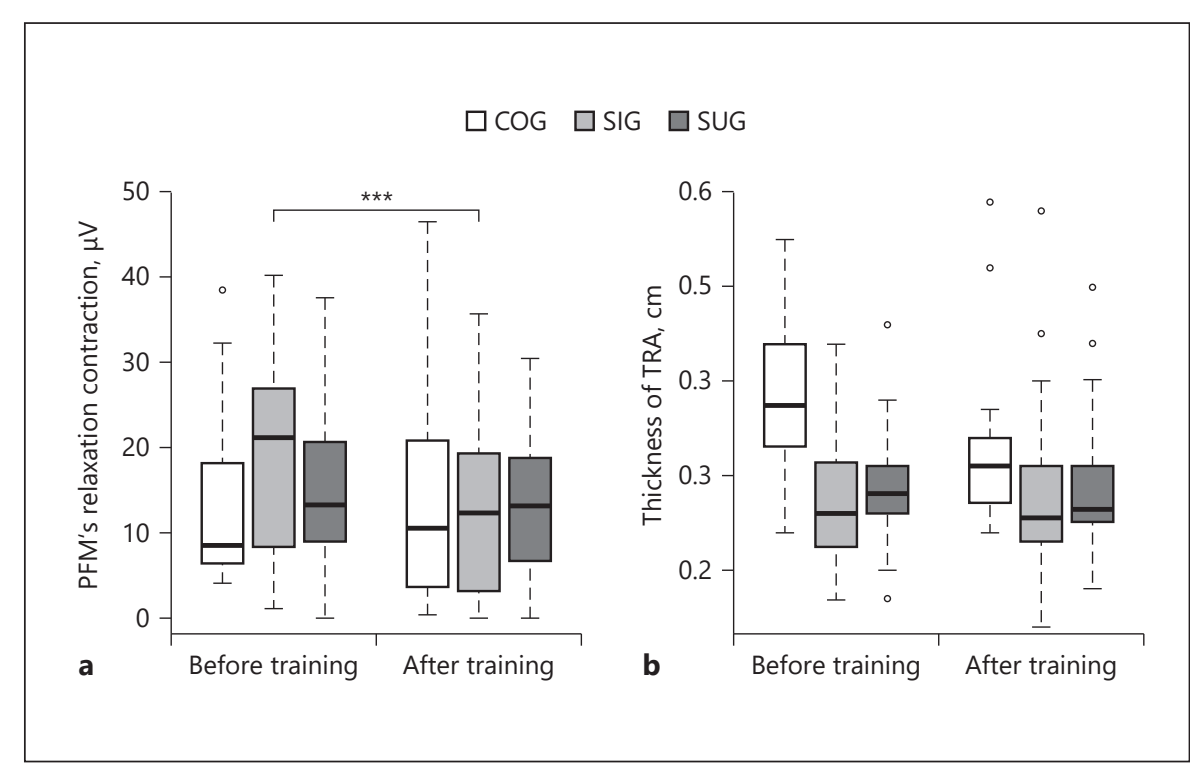

Thompson et al. [25] suggested careful monitoring of Sapsford's complex rehabilitation training because abdominal muscles are more active than PFMs in symptomatic women. These results suggest that a coordinated approach involving both deep abdominal muscles and PFM is necessary.

The correct breathing technique is very important in PFM-T. The diaphragm is a respiratory muscle participating in the stabilization of the lumbar spine by enhancing abdominal pressure [26], which in turn stabilizes the lumbar spine. Hodges et al. [27] reported synergism between the diaphragm and the TRA muscle. We also utilized this synergism by forced exhalation in our study.

Neumann and Gill [6] suggested that the activation of deep abdominal muscles is essential for an effective contraction of the PFM because their continent participants were unable to contract the PFM effectively while maintaining relaxation of the TRA muscle and the IO. Similarly, Madill and McLean [22] found that the patterns of abdominal muscle activity appear to occur due to voluntary PFM contractions in healthy continent women.

As a representation of vaginal closure force, the isometric contraction was considered to be greater in the supine than in the standing position. Subsequent studies however have determined that women are able to perform equally strong PFM contractions in either body positions $[7,8,11]$.

The PFM is a striated muscle, with two-thirds of its fibres being type I (slow-twitch fibres), responsible for the resting tone of the levator muscle and one-third of its fibres type II (fast-twitch fibres), responsible for sudden, fast but powerful contractions. The activity of the slow fibres is necessary for the resting potential and the retention of urine and stool, while the fast fibres are responsible for resistance during sudden abdominal pressure [28]. We monitored the activity of these 2 fibre types (retention and rapid function) in our study with vsEMG measurements and trained both types in weaker and stronger PFM-T.

The holding function of the PFM is important for the patients - that is, they need to be able to get to the toilet in time before their urine starts leaking. The quality of breathing plays an important role in PFM training. In our study, we activated the TRA muscle and voluntary contraction of the PFM with strong exhalation techniques during both measurements and training. Using biofeedback (ultrasound investigations and vsEMG curves), we visualized the co-contraction of the 2 muscles. Our vsEMG findings indicate that the static isometric contraction force of the PFM increased significantly in both study groups, and the holding time of static isometric contraction of the PFM increased slightly, with forced exhalation. In the COG, the static strength of the PFM increased slightly, but this increase was not significant and was characterized by a short retention time. The COG claimed that this parameter decreased significantly because, during our study, they had spent much more time in a sitting position and were therefore in an enforced inactive lifestyle. Another study obtained similar results for musculus multifidus [29].

Hung et al. [14] investigated the effects of combining voluntary PFM and deep abdominal muscle training in different body positions. Chmielewska et al. [11] mea- 
sured the rapid activity of the PFM with vsEMG in the supine position and in the sitting position, finding no significant differences between the PFM average peak amplitudes in the investigated positions, while we found these values to be significantly improved in the supine position. In our study, only in the supine position did the strength and repetition of dynamic fast contractions increase or significantly - in the sitting position - changes in dynamic fast contractions were not significant. The fast activity of the PFM is responsible for resistance during sudden abdominal pressure. In the COG, dynamic strength and repetition decreased and did not manifest significant changes.

Capson et al. [8] and Chmielewska et al. [11] measured the relaxation tone of the PFM in the supine and sitting positions. They deduced that gravity forces increase the pressure on the PFM in the vertical position, increasing its tone and leading to a higher resting activity of the PFM in the sitting position than in the supine position. During our measures, the SIG participants found it easier to relax in the horizontal position. Based on our results, practising relaxation exercises can be beneficial not only in the horizontal position but also in the vertical position. Improvement in the relaxation ability of the PFM is also a beneficial result because, especially during urination, for a healthy, normal urination mechanism, it is necessary to consciously relax the PFM. The relaxation state of the PFM improved significantly due to gravity forces only in the SIG.

We observed that the conditioning capabilities of the PFM improved in the SUG group during the training ( $\mathrm{cP}$ FM-T). The ability to sustain isometric contractions improved significantly, while holding time also improved, but this improvement was not significant. There was also a significant improvement in dynamic endurance and repetitions. Therefore, we recommend a more intense strengthening of the TRA muscle in the horizontal body position.

In the case of the SIG, the maximal isometric contraction of the PFM till fatigue also improved significantly, together with the holding time, but the latter improvement was not significant. Thus, even more intense TRA muscle activation is required in the vertical position as a reliance on the enhanced gravitational forces caused by the lumbopelvic posture or on the activated TRA muscle induced by strong exhalation is insufficient to induce the necessary changes in the functioning of the TRA muscle.

In the case of the COG, all parameters decreased by reason of sedentary lifestyle. We also established that young nulliparous women might also be affected by urinary leakage (27\%), as confirmed by the results of Haslam [1].
In the case of the SUG group, both training exercises and measurements were performed in the supine position, whereas the SIG group participants were measured in the lying position but performed all the exercises in the sitting position. We recommend that during $\mathrm{CPFM}-\mathrm{T}$, isometric and relaxation tasks should be performed both in the supine and sitting positions.

According to Sapsford [5], the antigravity posture requires more intense PFM activity. Furthermore, Chmielewska et al. [11] reported a significant difference between the sustained 1-min contraction of the PFM in the supine position and sitting position, while we found that the maximal isometric sustained contraction of PFM till fatigue significantly improved both in the supine position and sitting position.

Group training and individual training, according to recent research, are equally effective, and group training is more cost-effective without limitation [30]. Furthermore, due to differences in knowledge and behaviour, physiotherapist-guided training can help and motivate patients in persistent PFM-T [31].

\section{Statement of Ethics}

The protocol and consent forms were approved by the Ethics Committee of the Hungarian National Healthcare Service Center. The title of the ethics approval: Non-interventional study "Effect of PFM on Urinary Incontinence and Sexual Quality of Life." The number of the ethics approval: 019234/2014/OTIG Registered 7 April 2014. The name of the Ethics Committee: Scientific and Research Ethics Committee of the Health Science Council. The address of the Ethics Committee: Hungary, 1,051 Budapest, Zrínyi street 3. Phone number: +36(1)8869329. E-mail: amd@ogyei.gov. hu. All participants provided written informed consent. Trial registration: This study was registered in the Hungarian National Healthcare Service Center: 019234/2014/OTIG (registered 7 April 2014). Modification: 096623-002/2015/OTIG (registered 11 November 2015), https://www.aeek.hu. The study was retrospectively registered in ClincalTrials.gov (NCT04577872; registered 22 September 2020). https://clinicaltrials.gov.

\section{Conflict of Interest Statement}

The authors have no conflict of interests.

\section{Funding Sources}

The project has been supported by the European Union, cofinanced by the European Social Fund (EFOP-3.6.1-16-201600008). The role of the funding body in the design of the study and collection, analysis, and interpretation of data and in writing the manuscript should be declared. 


\section{Author Contributions}

All authors have read and approved the manuscript. T.M. was involved in the project development, study design, ultrasound investigation, data collection, manuscript writing, and editing; A.D. took part in project development, study design, and manuscript revision; M.S. contributed to data collection and statistical analysis; A.S. contributed to the study design, ultrasound investigation, manuscript writing, editing, and revision; J.B. was involved in project development and manuscript revision.

\section{Data Availability Statement}

The data that support the findings of this study are available on request from the corresponding author (A.S.) or the first auhtor (T.M.).

\section{References}

1 Haslam J. The prevalence of stress urinary incontinence in women. Nurs Times. 2004; 100(20):71-3.

2 Kegel AH. Progressive resistance exercise in the functional restoration of the perineal muscles. Am J Obstet Gynecol. 1948;56(2): $238-48$.

3 Dumoulin C, Hay-Smith J. Pelvic floor muscle training versus no treatment, or inactive control treatments, for urinary incontinence in women. Cochrane Database Syst Rev. 2010; 20(1):CD005654.

4 Thüroff JW, Abrams P, Andersson KE, Artibani W, Chapple CR, Drake MJ, et al. EAU guidelines on urinary incontinence. Eur Urol. 2011;59:387-400

5 Sapsford RR. The pelvic floor. A clinical model for function and rehabilitation. Physiotherapy. 2001;87:620-30.

6 Neumann P, Gill V. Pelvic floor and abdominal muscle interaction: EMG activity and intra-abdominal pressure. Int Urogynecol J Pelvic Floor Dysfunct. 2002;13(2):125-32.

7 Madill SJ, McLean L. Quantification of abdominal and pelvic floor muscle synergies in response to voluntary pelvic floor muscle contractions. J Electromyogr Kinesiol. 2008; 18(6):955-64

8 Capson AC, Nashed J, Mclean L. The role of lumbopelvic posture in pelvic floor muscle activation in continent women. J Electromyogr Kinesiol. 2011;21(1):166-77.

9 Sapsford RR, Richardson CA. Stanton WR Sitting posture affects pelvic floor muscle activity in parous women: an observational study. Aust J Physiother. 2006;52(3):219-22.

10 Sapsford RR, Richardson CA, Maher CF, Hodges PW. Pelvic floor muscle activity in different sitting postures in continent and incontinent women. Arch Phys Med Rehabil. 2008;89(9):1741-7.

11 Chmielewska D, Stania M, Sobota G, Kwasna K, Blaszczak E, Taradaj J, et al. Impact of different body positions on bioelectrical activity of the pelvic floor muscles in nulliparous continet women. BioMed Res Int. 2015.

12 Bø K, Mørkved S, Frawley H. Sherburn M Evidence for benefit of transversus abdominis training alone or in combination with pelvic floor muscle training to treat female urinary incontinence: a systematic review. Neurourol Urodyn. 2009;28:368-73.

13 Nie XF, Ouyang YQ, Wang L, Redding SR. A meta-analysis of pelvic floor muscle training for the treatment of urinary incontinence. Int J Gynaecol Obstet. 2017;138(3):250-5.

14 Hung $\mathrm{H}-\mathrm{C}$, Hsiao S-M, Chih S-Y, Lin $\mathrm{H}-\mathrm{H}$, Tsauo J-Y. An alternative intervention for urinary incontinence: retraining diaphragmatic, deep abdominal and pelvic floor muscle function coordinated function. Man Ther. 2010;15:273-9.

15 Sriboonreung T, Wongtra-ngan S, Eungpinichpong W. Laopaiboon M Effectiveness of pelvic floor muscle training in incontinent women at Maharaj Nakorn Chiang Mai Hospital: a randomized controlled trial. J Med Assoc Thai. 2011;94:1-7.

16 Bø K. Finckenhagen HB Is there any difference in measurement of pelvic floor muscle strength in supine and standing position? Acta Obstet Gynecol Scand. 2003;82:1120-4.

17 Da Roza T, de Araujo MP, Viana R, Viana S, Jorge RN, Bø K, et al. Pelvic floor muscle training to improve urinary incontinence in young, nulliparous sport students: a pilot study. Int Urogynecol J. 2012;23(8):106973.

18 Bø K, Kvarstein B, Hagen R, Larsen S. Pelvic floor muscle exercise for the treatment of female stress urinary incontinence: validity of vaginal pressure measurements of pelvic floor muscle strength and the necessity of supplementary methods for control of correct contraction. Neurourol Urodyn. 1990;9: 479-87.

19 Whittaker JL, Warner MB, Stokes M. Comparison of the sonographic features of the abdominal wall muscles and connective tissues in individuals with and without lumbopelvic pain. J Orthop Sports Phys Ther. 2013;43(1): 11-9.

20 Carrière B. Sensory awareness-feeling the pelvic floor. Carrière B, editor. Fitness for the pelvic floor. 1st ed. Stuttgart, New York: Georg Thieme Verlag; 2002. p. 24-30.

21 Shamsi M, Mirzaei M, HamediRad M. Comparison of muscle activation imbalance fol- lowing core stability or general exercises in nonspecific low back pain: a quasi-randomized controlled trial. BMC Sports Sci Med Rehabil. 2020;12:24. eCollection 2020.

22 Madill SJ, McLean L. Relationship between abdominal and pelvic floor muscle activation and intravaginal pressure during pelvic floor muscle contractions in healthy continent women. Neurourol Urodyn. 2006;25:722-30.

23 Sapsford R. Rehabilitation of pelvic floor muscles utilizing trunk stabilization. Man Ther. 2004;9:3-12.

24 Dumoulin C, Glazener C, Jenkinson D. Determining the optimal pelvic floor muscle training regimen for women with stress urinary incontinence. Neurourol Urodyn. 2011; 30:746-53.

25 Thompson JA, O'Sullivan PB, Briffa NK, Neumann P. Differences in muscle activation patterns during pelvic floor muscle contraction and Valsalva manouevre. Neurourol Urodyn. 2006;25(2):148-55.

26 Kapandji IA. The lumbar spine. In. Kapandji IA, editor. The physiology of the joints trunk and the vertebral column. London: Churchill Livingstone; 2008. p. 84-141.

27 Hodges PW, Butler JE, Mckenzie DK, Gandevia SC. Contraction of the human diaphragm during rapid postural adjustments. J Physiol. 1997;505(Pt 2):539-48.

28 Gosling JA, Dixon JS, Critchley HO, Thompson SA. A comparative study of the human external sphincter and periurethral levator ani muscles. Br J Urol. 1981;53:35-41.

29 Finta R, Nagy E, Bender T. The effect of diaphragm training on lumbar stabilizer muscles: a new concept for improving segmental stability in the case of low back pain. J Pain Res. 2018;11:3031-45.

30 Dumoulin C, Morin M, Mayrand MH, Tousignant M. Abrahamowicz M Group physiotherapy compared to individual physiotherapy to treat urinary incontinence in aging women: study protocol for randomized controlled trial. Trials. 2017;18(1):544.

31 Hay-Smith EJ, McClurg D, Frawley H. Dean SG Exercise adherence: integrating theory, evidence and behavior change techniques. Physiotherapy. 2016;102(1):7-9. 